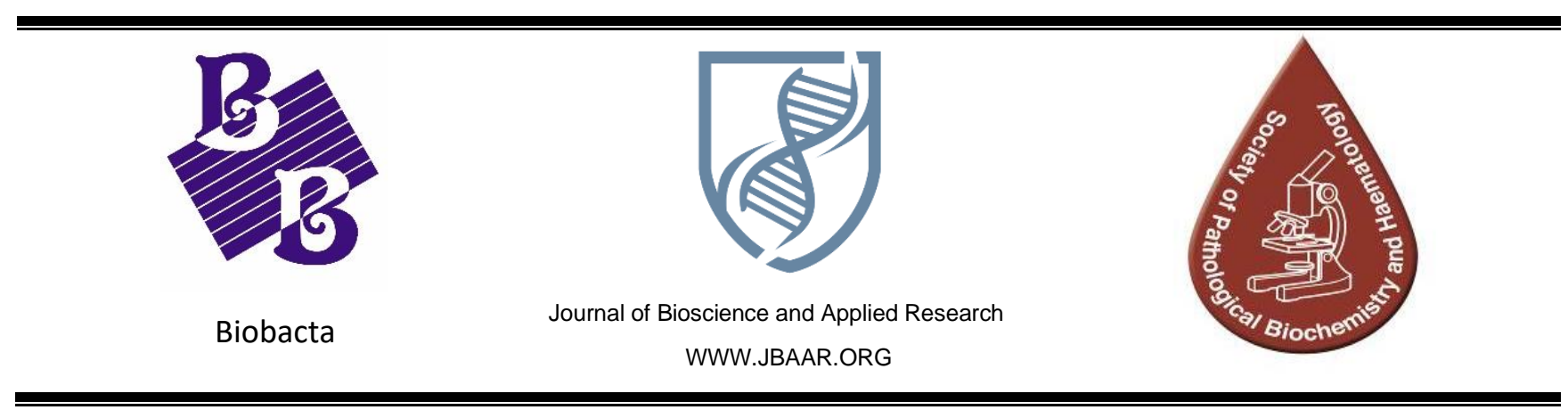

\title{
Schistosomiasis haematobium Prevalence and Risk factors in EL-Fayoum Governorate, Egypt
}

\author{
Ahmed AbdelhalimYameny \\ Society of Pathological Biochemistry and Hematology, Egypt \\ Ahmed A. Yameny (Email: dr.ahmedyameny@yahoo.com) \\ DOI: 10.21608/jbaar.2017.126150
}

\begin{abstract}
Schistosoma haematobium infection is an important water-borne disease in Egypt, its prevalence decreased to less than $0.2 \%$ (2016). There are more than 300 villages in Egypt with a prevalence of more than $3 \%$, especially among school children, Which require prolonged treatment and continuous examinations, The Ministry of Health and Population in Egypt has announced the start of a campaign to confirm the final elimination of schistosomiasis by 2020, this study was conducted to detect the prevalence of Schistosoma haematobium and risk factors among 1000 patients attending Ministry of Health Laboratory Centers, in El-Fayoum Governorate by microscopic examination in rural and urban areas, the prevalence among study patients was $7.9 \%$, an interview questionnaire was designed to obtain demographic data and risk factors, such as residential status, age, water contact activity, occupation, and education level, the higher percentage of infection was $10.3 \%$ among 11-21 years group, infection among males was higher than that among females (9.0\% vs.6. $8 \%$ respectively). The percentage of infection was $11.2 \%$ for rural residence compared to $2.4 \%$ for urban residence, regarding occupation the higher percentage of infection was $27.6 \%$ among farmers and /or fishermen. According to contact with canal water, the percentage of infection among those who had water contact was $16.9 \%$ and only $0.9 \%$ for people with no water contact. This study, therefore, recommended that a schistosomiasis control program in these infected areas should be done to educate the population on risk factors that predispose an individual to urinary schistosomiasis and the need for proper control of snails the intermediate host besides treatment of infected people.
\end{abstract}

Keywords: Schistosoma haematobium, Egypt, prevalence, risk factors, final elimination, El-Fayoum

\section{Introduction}

Schistosomiasis is a disease of poverty. It gives rise to much suffering and death, it also contributes to the perpetuation of poverty by impairing the cognitive performance, growth of children, reducing the work capacity, and productivity of adults ${ }^{(1)}$. It was recorded that $85 \%$ of all schistosomiasis infections are found in sub-Saharan Africa, mostly among poor people who live in remote areas without access to health services, safe water, sanitation, and education $^{(2)}$. Contact of the definitive host with cercaria infested water is essential for humans to be infected. Surface water such as rivers, ponds or lakes, streams, and irrigation canals are the usual sources of infection ${ }^{(3)}$. Three categories of exposure to Schistosome infected 
water are; occupational exposure during agriculture related jobs, recreational exposure during bathing,playing,or swimming in canal water and domestic exposure while washing clothes and utensils in canal water ${ }^{(3,4)}$. In Africa, schistosomiasis reported near to 300,000 deaths per year ${ }^{(5)}$. In Egypt, Schistosomiasis was the major public health problem for several decades due to high prevalence and morbidity especially among rural populations, which caused by both S.haematobium and S.mansoni ${ }^{(6)}$. This infection has been recorded in Egypt from early pharaonic times (3200 B.C.) by the demonstration of circulating Schistosome antigens and Schistosome eggs in mummies ${ }^{(7)}$. After the discovery of the parasite's life cycle in1915, Egypt started to fight against the disease, a changing pattern of schistosomiasis infection has been observed as S.haematobium was decreasing from $48 \%$ in 1935 to less than $0.2 \%$ in 2016, and S.mansoniw as decreasing from $32 \%$ in 1935 to less than $0.2 \%$ in $2016^{(8)}$.

The World Health Assembly in 2012 called, for the first time, for Schistosoma transmission interruption wherever possible. recognition of 'persistent hot spots' among treated areas within control districts indicates that we need to break both human-to-snail and snail-to-human transmission to fully prevent new infections, to reach elimination of Schistosoma ${ }^{(8)}$. The Ministry of Health and Population in Egypt has announced the start of a campaign to confirm the final elimination of schistosomiasis by 2020 after the ministry has achieved success in reducing the prevalence of schistosomiasis to about $0.2 \%$ by the end of 2016. Treatment by targeting 6 million schoolchildren and citizens at a total cost of 40 million pounds including pesticide, praziquantel and covering the cost of health teams and watercourse treatment in cooperation with the World Health Organization $^{(9)}$. There are more than 300 villages in Egypt with a prevalence of more than 3\%, especially among school children, Which require prolonged treatment and continuous examinations ${ }^{(9)}$.
A better understanding of risk factors for schistosomiasis is important in controlling the disease, we, therefore, aimed to investigate the S.haematobium risk factors and prevalence, the present study used both the microscopic examination of urine to detect the infection rate and Questionnaires to study the risk factors, EL-Fayoum Governorate is supplied by water through a single canal (Bahr Youssef) from the Nile River, it has had the longest ongoing Schistosomiasis control program in Egypt.

\section{Research objective:}

Is to Estimate the Prevalence of Schistosoma haematobium and Risk factors in EL-Fayoum Governorate, Egypt

\section{Material and methods}

\subsection{Study population and ethical consideration}

This study included 1000 patients attending the Ministry of health laboratory centers, in El-Fayoum Governorate, these study subjects were randomly selected irrespective of the age-group and both genders were included. All the studied population was informed about the purpose of sample collection and their consent was obtained. Patients were free to refuse sample collection.

\subsection{Study design}

This research is a cross-sectional study designed to determine infection prevalence and risk factors for transmission of urinary schistosomiasis in the study population. This study was targeting customers who came for laboratories of health centers for urine analysis.

\subsection{Collection and processing of urine samples}

Clean specimen bottles were labeled with the needed information and issued to the participating individuals whose informed consent was sought earlier, each patient was 
given a wide mouth screw-capped container into which to void urine. This was carried out between 10.00 am and $2.00 \mathrm{pm}$ when the ova count of S.haematobium is expected to be at its peak $^{(10)}$. In this study, urinary schistosomiasis was defined as the presence of ova of S.haematobium in the urine.

\subsection{Urine microscopy}

Urine examination was carried out in the laboratory of health centers and by the researcher. Urine samples were examined for the presence of S.haematobium eggs as in the sedimentation method of Cheesbrough (2006) (11). Each urine sample was mixed thoroughly with a glass rod and two samples were taken for each $10 \mathrm{ml}$ urine, one sample for sedimentation centrifugation and the other $10 \mathrm{ml}$ urine sample for the Nuclepore membrane filtration technique. The first $10 \mathrm{ml}$ transferred into a centrifuge tube and centrifuged at $2000 \mathrm{rpm}$ for 5 minutes at room temperature. The supernatant was then discarded and sediment was transferred to a microscope glass slide and covered with a coverslip. A drop of Lugol's Iodine was added onto the coverslip before the examination. Examination of the entire sediment was carried out using the $\mathrm{x} 10$ objective of a compound light microscope.

The second $10 \mathrm{ml}$ urine sample was examined using the Nuclepore membrane filtration technique for S.haematobium eggs detection as in the method of Cheesbrough (2009) ${ }^{(12)}$.

\subsection{Administration of Questionnaires}

Table (1): Percentages of S.haematobium infection among the study sample according to urine examination by direct parasitologic techniques.

\begin{tabular}{|l|c|c|c|l|l|}
\hline \multirow{2}{*}{\begin{tabular}{|} 
Direct \\
ParasitologicTechniques
\end{tabular}} & \multicolumn{4}{|c|}{ S.haematobium } & \multirow{3}{*}{ Total } \\
\cline { 2 - 5 } & \multicolumn{2}{|c|}{ Negative } & \multicolumn{2}{c|}{ Positive } \\
\cline { 2 - 5 } & NO. & $\%$ & NO. & $\%$ & \% \\
\hline Sedimentation & 936 & 93.6 & 64 & 6.4 & 1000 \\
\hline Nucleporefilteration & 923 & 92.3 & 77 & 7.7 & 1000 \\
\hline Both techniques & 921 & 92.1 & 79 & 7.9 & 1000 \\
\hline
\end{tabular}

Sedimentation with a sensitivity of $81 \%$ while nuclepore filtration sensitivity was $97.5 \%$
An interview questionnaire was designed for the research and administered to the participating patients of different age groups and both sexes, who had health center customers to provide their urine for the study. The questionnaires contained the necessary questions intended to obtain demographic data and risk factors relevant to that research objective, such as residential status, age, water contact activity, occupation, and education level ${ }^{(13)}$. A total of 1000 questionnaires were administered to the study subjects.

\subsection{Statistical analysis}

Results were collected, coded, tabulated, and analyzed through computer facilities using statistical methods and relationships with age, sex, and other risk factors for Schistosoma infection. S.haematobium infection was defined as any number of eggs greater than zero found in $10 \mathrm{ml}$ of urine, was performed to assess the association between the status of S.haematobium infection and the associated risk factors.

\section{Results}

Percentages of S.haematobiuminfection: Table (1) shows percentages of S.haematobium infection as detected by different direct parasitologic techniques. The sedimentation technique revealed a percentage of $6.4 \%$ which increased to $7.7 \%$ using the nuclepore filtration technique. Prevalence increased to $7.9 \%$ when the results of both techniques were considered. 
Distribution of S.haematobium infection among the study sample:

Table (2) displays a distribution of S.haematobium infection among the study sample according to some risk factors. The total study sample was stratified according to age into four categories. The higher percentage of infection was $10.3 \%$ among the $11-21$ years group. It was $8.8 \%$ among those less than 11 years and $5.2 \%$ for those between 21 and 36 years. Only $2 \%$ belonged to the age group between 36 and 70 years. Young age group was a risk factor for S.haematobium infection it was 4.6 times and 5.5 times more in the $<11$ years group and 11-21 years group respectively than the older group of (36- 70 years old).GMEC increased with age from $6.8 \mathrm{eggs} / 10 \mathrm{ml}$ urine in age group $<11$ years to $24.5 \mathrm{eggs} / 10 \mathrm{ml}$ urine in the age group 36-70 years, except in 21-36 years group in which it decreased to $4.4 \mathrm{egg} / 10$ $\mathrm{ml}$ urine.

According to gender, the distribution of S.haematobium infection among males was higher than that among females $(9.0 \%$ vs.6. $8 \%$ respectively). However, the difference was not statistically significant $(\mathrm{O} . \mathrm{R}=1.3$ and $\mathrm{CL}=$ 0.851- 2.158). Also, GMEC for males was 9.08 eggs/10 $\mathrm{ml}$ urine higher than that for females (6.39 eggs/10 $\mathrm{ml}$ urine).

Residents living in rural areas showed 4.7 times the risk of infection compared to urban areas. The percentage of infection was $11.2 \%$ for rural residence compared to $2.4 \%$ for urban residents. The difference was statistically significant $(95 \% \mathrm{CL}=0.096-0.395)$. Also, GMEC was $8.93 \mathrm{egg} / 10 \mathrm{ml}$ urine among residents of rural areas and only $2.84 \mathrm{egg} / 10 \mathrm{ml}$ urine for those living in urban areas.

Regarding occupation, the higher percentage of infection was $27.6 \%$ among farmers and /or fishermen. It was only $5.4 \%$ among students, and $3.7 \%$ among housewives. It is 9.8 times more for farmers and /or fishermen than housewives, (95\% CL for the farmer and/ or fishermen was high it 3.619- 26.569), Also GMEC was $9.8 \mathrm{egg} / 10 \mathrm{ml}$ urine among farmer and/ or fishermen, 8.06 among students, 4.74 egg/10 ml urine among housewives and 6.26 among others.

Considering education, $13.5 \%$ of illiterate or read and write were infected. The percentage ofS.haematobium infection was only $5.1 \%$ among those having primary /or preparatory educations were infected, and only $4.2 \%$ of those who had a secondary education were infected. No infection was diagnosed among those having university or higher education. The risk of infection was 2.9 and 3.5 times more for preschool and illiterate/read and write respectively than for those having a secondary education. Also, GMEC was $8.28 \mathrm{egg} / 10 \mathrm{ml}$ urine for the secondary group and nearly equal for other groups (ranging between $7.4 \mathrm{egg} / 10$ $\mathrm{ml}$ urine to $7.9 \mathrm{egg} / 10 \mathrm{ml}$ urine).

According to contact with canal water, the percentage of infection among those who had water contact was $16.9 \%$ and only $0.9 \%$ for people with no water contact. Risk of infection was 22.5 times more for those with water contact, (95\% CL for water contact was 9.02756.302). Also GMEC was $8.08 \mathrm{egg} / 10 \mathrm{ml}$ urine among people who had water contact and 2.45 egg/10 ml urine for those with no water contact. 
Table (2): Distribution of S.haematobium infection among the study sample:

\begin{tabular}{|c|c|c|c|c|c|c|}
\hline \multirow{2}{*}{ Risk factors } & \multirow{2}{*}{$\begin{array}{l}\text { No. } \\
\text { examined }\end{array}$} & \multicolumn{2}{|c|}{ S. haematobium } & \multirow{2}{*}{ Odds ratio } & \multirow{2}{*}{$\begin{array}{c}95 \% \\
\text { Confidence limits }\end{array}$} & \multirow{2}{*}{$\begin{array}{l}\text { GMEC/ } \\
10 \mathrm{ml} \text { of } \\
\text { urine }\end{array}$} \\
\hline & & No. & $\%$ & & & \\
\hline \multicolumn{7}{|l|}{ 1-Age group in( years): } \\
\hline$<11$ & 319 & 28 & 8.8 & $4.667^{*}$ & $1.092-19.951$ & 6.81 \\
\hline |11- & 380 & 39 & 10.3 & $5.547^{*}$ & $1.316-23.383$ & 9.53 \\
\hline $21-$ & 193 & 10 & 5.2 & 2.650 & $0.569-12.338$ & 4.35 \\
\hline $36-70 ®$ & 99 & 2 & 2 & 1.0 & - & 24.49 \\
\hline \multicolumn{7}{|l|}{ Sex } \\
\hline Male & 513 & 46 & 9.0 & 1.355 & $0.851-2.158$ & 9.08 \\
\hline Female ${ }^{\circledR}$ & 487 & 33 & 6.8 & 1.0 & - & 6.39 \\
\hline \multicolumn{7}{|l|}{ Residence } \\
\hline Urban ${ }^{\circledR}$ & 375 & 9 & 2.4 & 1.0 & - & 8.93 \\
\hline Rural & 625 & 70 & 11.2 & 4.67 & $0.096-0.395$ & 2.84 \\
\hline \multicolumn{7}{|l|}{ Occupation } \\
\hline Farmer and/or Fisherman & 98 & 27 & 27.6 & $9.811^{*}$ & $3.619-26.569$ & 9.80 \\
\hline Student & 521 & 28 & 5.4 & 1.465 & $0.555-3.870$ & 8.06 \\
\hline House wife ${ }^{\circledR}$ & 134 & 5 & 3.7 & 1.0 & - & 4.74 \\
\hline Others & 247 & 19 & 7.7 & 2.150 & $0.784-5.894$ & 6.26 \\
\hline \multicolumn{7}{|l|}{ Education } \\
\hline Preschool & 96 & 11 & 11.5 & 2.976 & $0.997-8.885$ & 7.44 \\
\hline Illiterate + read and write & 282 & 38 & 13.5 & $3.582^{*}$ & $1.374-9.340$ & 7.85 \\
\hline Primary + preparatory & 486 & 25 & 5.1 & 1.247 & $0.467-3.329$ & 7.93 \\
\hline Secondary ${ }^{\circledR}$ & 120 & 5 & 4.2 & 1.0 & - & 8.28 \\
\hline University or higher & 16 & 0 & 0.0 & - & - & 7.84 \\
\hline \multicolumn{7}{|l|}{ Exposure to canal water } \\
\hline Yes & 439 & 74 & 16.9 & $22.545^{*}$ & $9.027-56.302$ & 8.08 \\
\hline No & 561 & 5 & 0.9 & 1.0 & - & 2.45 \\
\hline
\end{tabular}

(:) reference group 
Figure (1): Distribution of urinary Schistosomiasis according to age groups

\section{Distribution of urinary Schistosimiasis according to age groups}

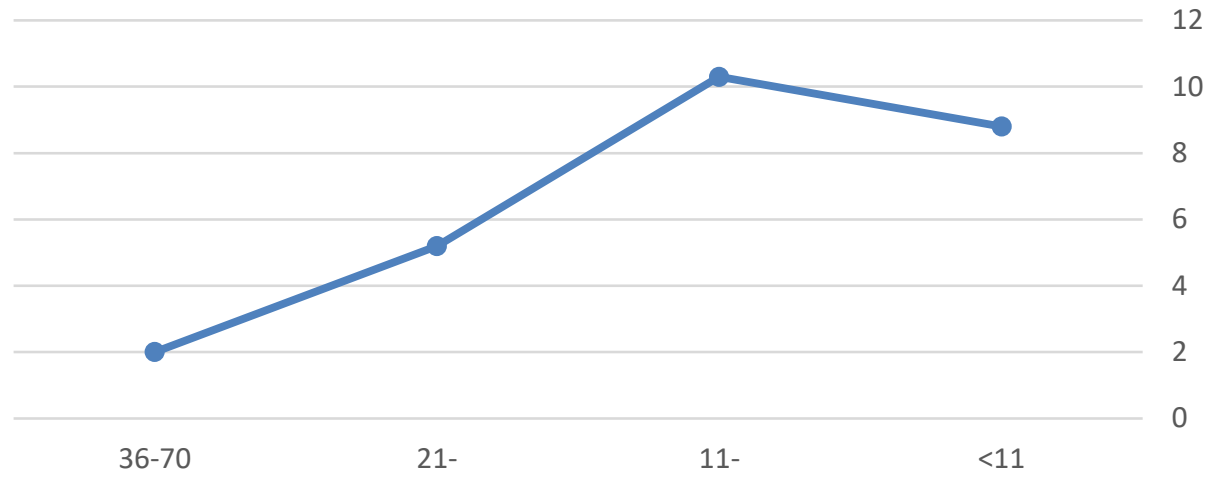

Figure (2): Distribution of urinary Schistosomiasis according to gender

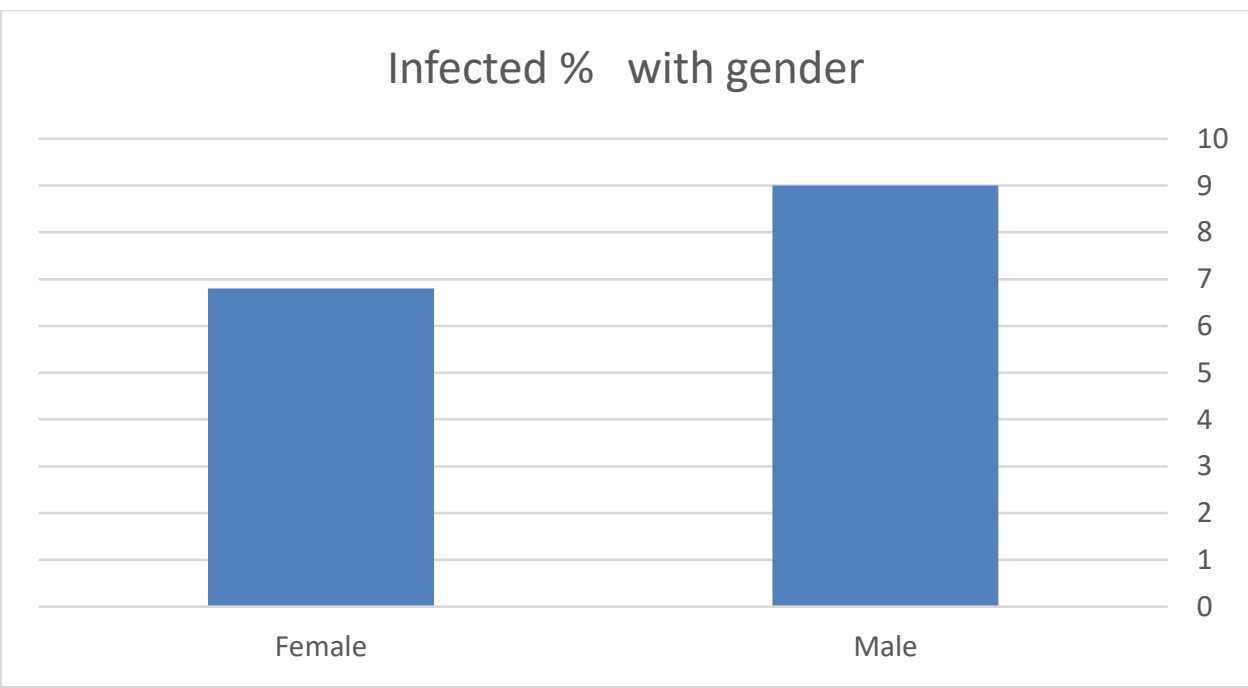

Figure (3): Distribution of urinary Schistosomiasis according to age Residence

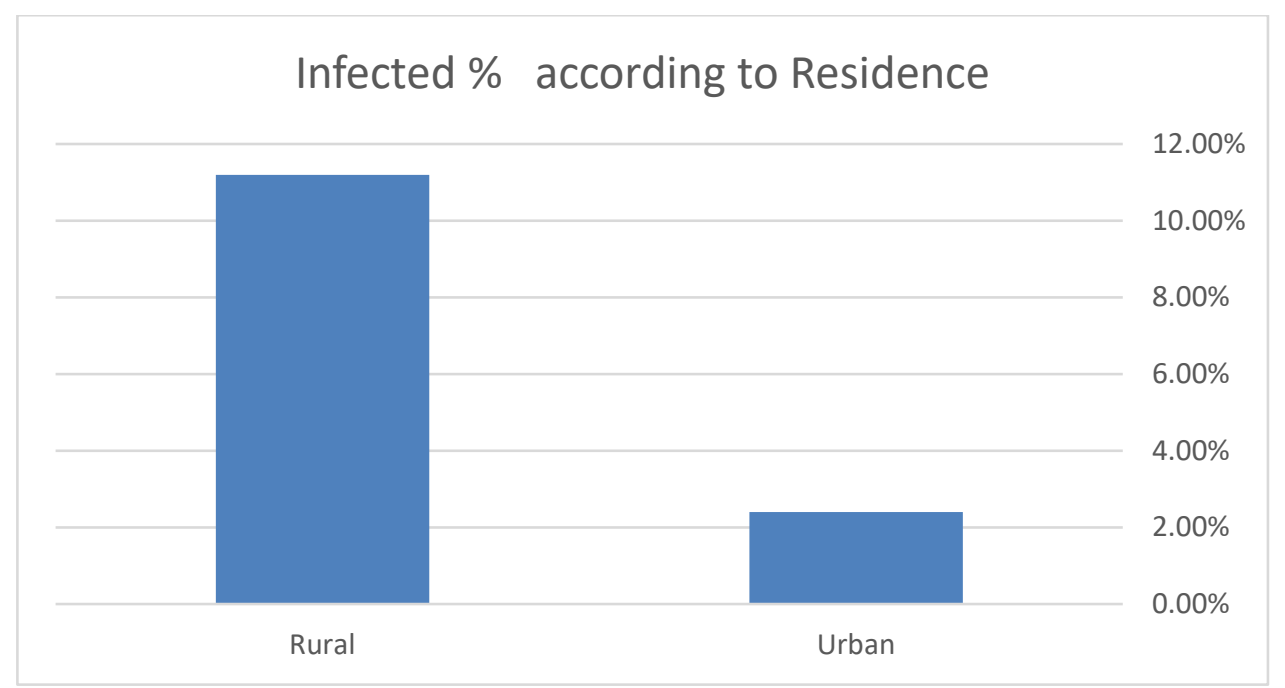


Figure (4): Distribution of urinary Schistosomiasis according to Occupation

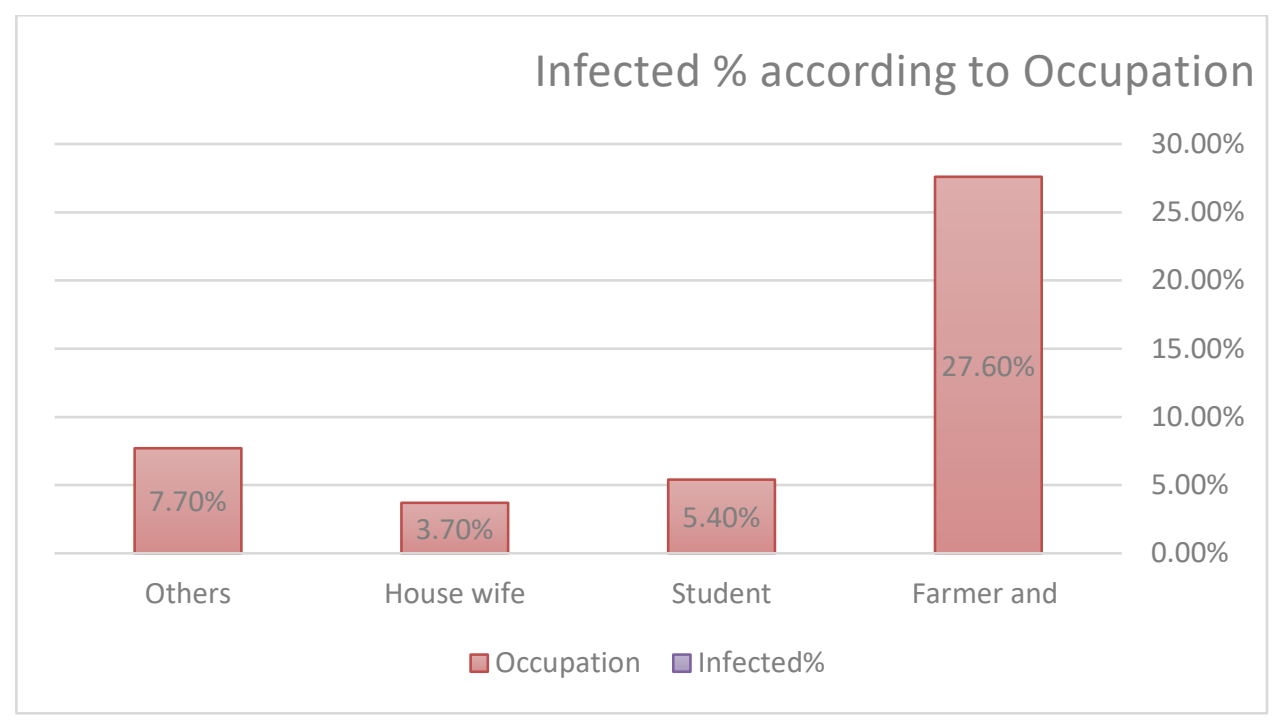

Figure (5): Distribution of urinary Schistosomiasis according to age Education

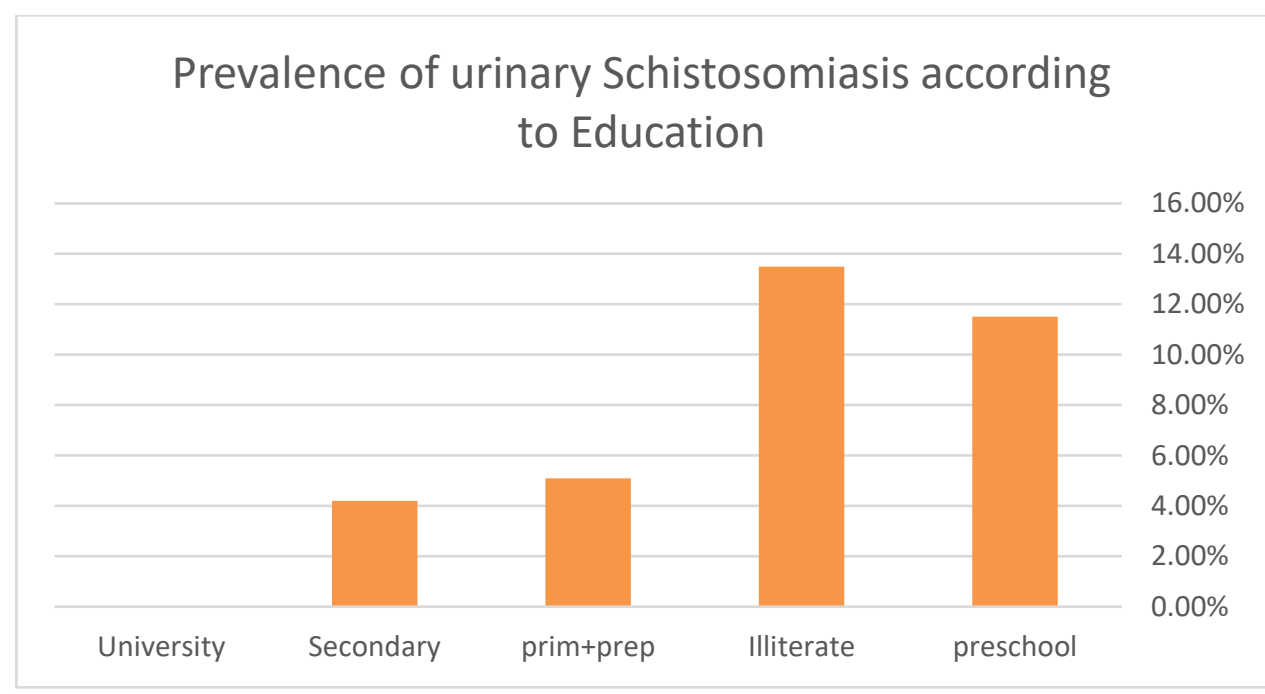

Figure (6): Distribution of urinary Schistosomiasis according to Exposure to canal water

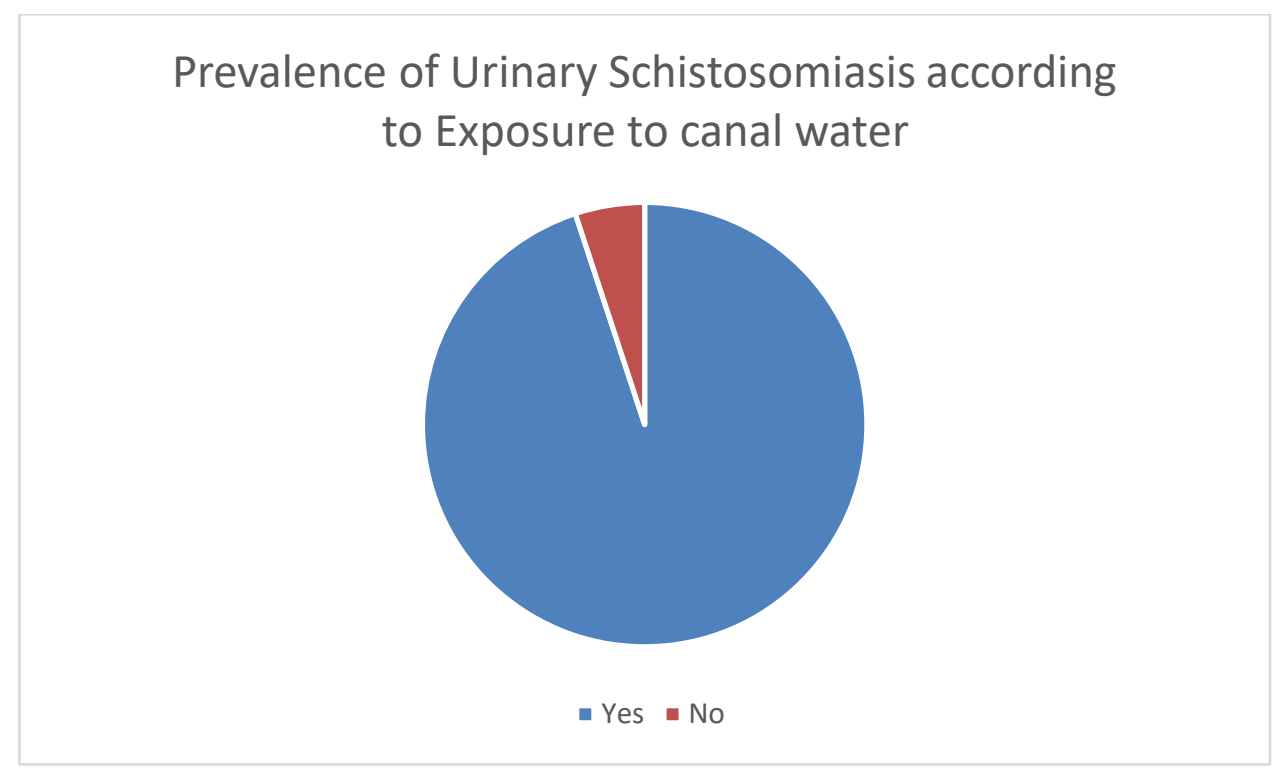




\section{Discussion}

The prevalence of S. haematobium among the study sample was $7.9 \%$ by both parasitological techniques sedimentation and nuclepore filtration(table1).

Risk factors of S. haematobium as revealed by the distribution of infection among the studied population sample (table2):

Age as a risk factor: This study revealed that the age group from 11-20 years showed the highest infection rate $(10.3 \%)$ followed by the group aged $<11$ years $(8.8 \%)$ and the least percentage of infection was among those aged 36 years and more. This finding was approved by other studies, Abdel-Wahab and Esmat, (2000), That reported followed by the age group in El-Fayoum also, the highest infection rate was age among the group 11-20 years $20.9 \%$ followed by the age group 0-10 ( $16.1 \%)^{(4)}$. Talaat and El-Ayat, et al, (1997). In a village in Giza, the prevalence of $S$. haematobium showed a peak at the age of 1014 years, it was $(35.9 \%)$ compared to infection in the village $(12.3 \%)^{(14)}$. Meanwhile, a study by Eric and Muchiri in coastal Kenya (2006), showed that the prevalence of infection among the age group 12-20 years was $15 \%$ compared with $27 \%$ for the 5-11 years old group. ${ }^{(15)}$ Also, a study by Kalu, KaluMong, et al, in Nigeria (2016) showed that the prevalence of infection was higher among children ages 11 to 15 years old $(22.22 \%)^{(16)}$. While a study by Kabuyaya et al, in South Africa (2017) showed aged 13 years were the most infected $(25 \%)^{(17)}$.

Concerning gender and its relation to infection, This study showed that the infection rate between males $(9.0 \%)$ is higher than among females (6. 8\%) (Abdel-Wahab 2000) also carried out an epidemiological study in ElFayoum and showed that the infection rate among males (16\%) was also higher than among females $(11.5 \%)^{(4)}$. Also, Talaat's (1997) study in Giza showed that the rate was (15.9\%) among males and (8.4\%) among females ${ }^{(14)}$. But a study carried out in Kenya for nine years (from 1984-1992) in most years females had a higher prevalence of infection than males, over the nine years of the study, except three years during which males had a higher prevalence than females (e.g., 1988, 1989, and $1990)^{(15)}$.KaluMong et al, in Nigeria (2016)showed that the prevalence of infection was higher in males $(18.38 \%)$ than females $(10.08 \%)$. While a study by Kabuyaya et al, in South Africa (2017) showed females had a higher rate of S.haematobium infection $60.8 \%$ and males was $39.2 \%{ }^{(17)}$.

In our result Risk factors for $S$. haematobium infection in El Fayoum was an age less than 20 years and male gender and 
male bathing or children playing or swimming in canal water. Males who work in agriculture and fishing require water contact males are exposed to infection than females.

As regards residence and occupation this study showed that the infection rate in Rural areas $(11.2 \%)$ was higher than that of urban areas $(2.4 \%)$ also, the study showed the higher infection rate was among fisher + farmer (27.6\%), student $(5.4 \%)$, housewife $(3.7 \%)$ and others $(7.7 \%)$. Water activities as most people in villages work in agriculture and fishing in addition to other activities bathing, swimming, washing utensils, and clothes are more susceptible to infection, alsoKaluMong et al, in Nigeria (2016) showed that prevalence of urinary schistosomiasis concerning occupational status, farmers had the highest infection $19.27 \%$. Kabuyaya et al, in South Africa (2017) showed S.haematobium infection had a strong relationship with these factors: the main source of domestic water, bathing, and washing clothes at an open-source of water.

Regarding education, This study showed the highest infection rate was among illiterate + read and write $(13.5 \%)$ followed by preschool group $(11.5 \%)$ than primary preparatory (5.1\%). The result was among pupils of secondary schools (4.2)\% and followed by a university or higher 16 cases only examined with no infection with $S$. haematobium. following this, Husein et al, (1996) proved that children not attending school are at higher risk of schistosomiasis infection than those attending school ${ }^{(18)}$. This was explained by the fact that children not attending school spend more time playing in the water or working in agriculture, hence at greater risk of infection. Meanwhile, Talaat(1997), Hang and Manderson (1992). showed that literacy and school attendance were not related to infection ${ }^{(14,19)}$. while studies one in South Africa (2017), and another study in Ethiopia (2015), showed that $90.0 \%$ of those infected knew bilharzia where, although children were ware of the disease, the environment in which they lived remained a threat to them, they do not have a better option $^{(17,20)}$.

\section{Conclusion and Recommendations}

Based on the results of this study which showed that urinary schistosomiasis is prevalent in some areas in EL-Fayoum even though it is moderate, the infection depends on water contact activities with some risk factors as age, gender, education, residence, and occupation, this study, therefore, recommended that schistosomiasis control program in these infected areas should be done upon to educate the population on risk factors that predispose an individual to urinary schistosomiasis and the need of proper to control of snails the intermediate host beside treatment of infected people. 


\section{Conflict of interest}

There are no conflicts of interest.

\section{Financial support and sponsorship}

This research did not receive any specific grant from funding agencies in the public, commercial, or not-for-profit sectors.

\section{References}

1. WHO (2002): Helminth control in schoolage children a guide for managers of control programs, Geneva.

2. Watts S. The social determinants of schistosomiasis 2005, Geneva WHO TDR/ SWG/07.

3.http://www.dpd.cdc.gov/dpdx/HTML/Schisto somiasis.htm

4. Hammad TA, Gabr NS, Hussien MH, Arieby A, Shawky E, Strickland GT. Determinants of infection with schistosoma haemtobium using logistic regression. Am J Trop Med Hyg 1997;57(4): 464 - 468.

5. Hotez, P.J., Fenwick, A., 2009.

Schistosomiasis in Africa: an emerging tragedy in our new global health decade. PLoS Negl. Trop. Dis. 3 (9), e485. 29.

6. Youssef MM. Current situation and research needs for schistosomiasis control in Egypt (2005), Geneva, WHO Tor/ SWG/ 01, 2005.

7. Kolata G. Avoiding the schistosome's tricks. Science 1985; 277: 285-7.

8. Ahmed A. Yameny. J Biosci App Res The evolving schistosomiasis agenda 20172020 in Egypt: Moving from control toward final elimination 2017;3(2): 48- 54.

9. Data from the Ministry of health and population, Endemic disease control department. Annual Report on
Schistosomiasis Control, 2017.

10. Eyong ME, Ikepeme EE, Ekanem EE. Relationship between Schistosoma haematobium infection and urinary tract infection among children in South Eastern, Nigeria. Niger Postgrad Med 2008;15:89-93.

11. Cheesbrough M. District laboratory practice in tropical countries part 2 . United Kingdom: Cambridge University Press. Cambridge. 2006; 323-41.

12. Cheesbrough M. Laboratory-practice-intropical-countries-part-1. United Kingdom: Cambridge University Press. Cambridge. 2009;p.357

13. Ali MU, Ndams IS. Human Schistosoma infections: Intensity versus water contact patterns among irrigation communities in Kano State, Nigeria. Biological and Environmental Sciences Journal for the Tropics. 2013;10(3):120-124.

14. Talaat MM, El-Ayyat AA, Sayed HA, Meikheimer SH. Individual, household, and behavioral risk factors of schistosoma haematobium infection in an Egyptian village in Giza governorate. J Egypt Soc Parasitol 1997; 27(1): 211-221.

15. Satayathum SA, Muchiri EM, Ouma JH, Whalen $\mathrm{CC}$, and King $\mathrm{CH}$. Factors affecting infection or reinfection with schistosoma haematobium in Coastal Kenya: Survival analysis during a nineyear, school-based treatment program. Am J Trop Med Hyg .,75(1),2006, pp.8392.

16. Kalu, Kalu Mong, Samuel, Chikodi, Ihemanmaand Chioma Ada. Current Prevalence Status of Urinary Schistosomiasis among Children in Lokpanta Community, Abia State, Nigeria Galore International Journal of Health 
Sciences and Research Vol.1; Issue: 1; December 2016, pp. 35- 40.

17. Kabuyaya M, Chimbar M, Manyangadz, and Mukaratirwa S. Schistosomiasis risk factors based on infection status among school-going children in the Ndumo area, uMkhanyakude district, South Africa. Southern African Journal of Infectious Diseases 2017; 32(2):67-72.

18. Hussein MH, El-sayed MK, Talaat M, Elbadawy A, and Evans DB.(1996): WHO misses out with school based health programmes ? A study of schistosomiasis control in Egypt. Trans . Roy. Soc.Trop Med Hyg. 90: 362-365.
19. Huang Y, and Menderson L. (1992): Schistosomiasis and the social patterning of infection. Acta Tropica, 51: 125- 194.

20. Geleta S, Alemu A, Getie S, et al. Prevalence of urinary schistosomiasis and associated risk factors among Abobo Primary School children in Gambella Regional State, southwestern Ethiopia: a cross-sectional study. Parasites \& Vectors 2015;8(1):1106-14.

https://doi.org/10.1186/ s13071-0150822-5 